\title{
Modelagem Computacional de Árvores Circulatórias ${ }^{1}$
}

\section{Computational Modeling of Circulatory Trees}

\author{
Felippe Rocha Lobo de Abreu ${ }^{2}$ \\ Rafael Alves Bonfim de Queiroz ${ }^{3}$
}

DOI: $\underline{\text { https://doi.org/10.34019/2179-3700.2019.v19.29887 }}$

\begin{abstract}
Resumo
A principal motivação para a construção automática de modelos de árvores circulatórias in silico é a inviabilidade de ter dados anatômicos suficientes que permitam caracterizar em detalhe a estrutura geométrica de redes vasculares periféricas. A representação adequada destas redes é necessária para modelar adequadamente o efeito dos leitos periféricos na hemodinâmica do sistema arterial humano. Tendo isto em vista, este trabalho investiga o método Constrained Constructive Optimization (CCO) para construção de modelos de árvores circulatórias em domínios de perfusão tridimensionais.
\end{abstract}

Palavras-chave: Árvores circulatórias. Otimização. Anatomia vascular. Morfometria.

\begin{abstract}
The main motivation for the automatic construction of circulatory tree models is the lack of enough anatomical data to characterize in detail the geometric and topological structure of the peripheral vascular networks. The appropriate representation of these networks is necessary to model adequately the effect of the peripheral beds in the hemodynamics of the human arterial system. Taking this into account, this work investigates the Constrained Constructive Optimization (CCO) method in order to construct circulatory tree models in three-dimensional perfusion domains.
\end{abstract}

Keywords: Circulatory trees. Optimization. Vascular anatomy. Morphometry.

\section{INTRODUÇÃO}

A modelagem geométrica de árvores circulatórias é a base para simulações computacionais de fenômenos hemodinâmicos (QUEIROZ, 2013). Vários tipos de modelos têm sido empregados nestas simulações: (i) modelos a parâmetros condensados (MATES et al., 1988), (ii) modelos anatômicos (ONUKI; NITTA, 1993), (iii) modelos fractais (VAN BEEK et al., 1989) e (iv) modelos gerados pelo método Constrained Constructive Optimization (CCO) (SCHREINER; BUXBAUM, 1993; KARCH et al., 1999).

\footnotetext{
${ }^{1}$ Trabalho premiado no Seminário de Iniciação Científica da UFJF em 2016.

${ }^{2}$ PROBIC Júnior/FAPEMIG/UFJF.

${ }^{3}$ Departamento de Ciência da Computação, Instituto de Ciências Exatas, UFJF. rafael.bonfim@ice.ufjf.br
} 
Os modelos a parâmetros condensados reduzem as características de árvores circulatórias complexas a vários compartimentos, cada qual representando uma certa classe de vaso (artérias, arteríolas, capilares, vênulas, veias) caracterizada por uma resistência que modela a oposição à circulação do fluido e um capacitor que modela a complacência dos vasos, isto é, sua capacidade de deformação. Assim sendo, estes modelos ignoram os detalhes da estrutura geométrica, tanto no nível da descrição de cada vaso arterial como dos fenômenos hemodinâmicos inerentes a tal estrutura.

Os modelos anatômicos fornecem uma representação mais precisa de uma parte da estrutura de uma árvore circulatória, e, portanto, são capazes de descrever quantidades físicas com maior grau de detalhe, assim como abarcar cenários mais complexos de simulação devido ao fato de dispor da distribuição espacial dos vasos em um dado domínio de interesse.

Os modelos fractais são gerados com ou sem componentes randômicas. Eles reproduzem distribuições estatísticas de características morfométricas (raios, comprimentos e ângulos entre os vasos) de árvores circulatórias reais. No entanto, estes métodos desconsideram o arranjo dos vasos sanguíneos no espaço, o que resulta de interesse em situações de análise hemodinâmica de perfusão de tecidos e na dinâmica circulatória na presença de tumores, dentre outros exemplos.

Os modelos de árvores circulatórias gerados pelo método CCO podem ser tão detalhados como os modelos anatômicos e tão precisos como os modelos fractais. As estruturas conectiva e geométrica dos vasos destes modelos são otimizadas no próprio processo de construção em vez de serem pré-definidas ou desprezadas como ocorre no caso de modelos fractais. Diferentes de modelos anatômicos, os segmentos com seus respectivos raios e comprimentos emergem do procedimento do tipo CCO em vez de serem fornecidos diretamente. Além disso, as coordenadas espaciais que constituem os pontos extremos dos segmentos são dadas pelo método $\mathrm{CCO}$ em vez de serem negligenciadas como ocorre nos modelos a parâmetros condensados, ou irrelevantes como acontece com os modelos fractais.

Neste trabalho, o foco são os modelos gerados pelo método CCO. Pois, além deles incorporarem princípios de otimalidade, estes modelos reproduzem características importantes de árvores arteriais, tais como os raios dos segmentos (SCHREINER; BUXBAUM, 1993), os ângulos de ramificação (SCHREINER et al., 1994), bem como características globais relacionadas ao nível de pressão e de fluxo sanguíneo ao longo 
da árvore (SCHREINER et al., 1995). Por isso, os modelos de árvores circulatórias oriundos do $\mathrm{CCO}$ estão começando a ser empregados para estudar a hemodinâmica cerebral e cardíaca, dentre outras (BUI et al., 2010; KARCH et al., 1999; SCHREINER et al., 2006).

O restante deste trabalho está organizado como segue. Na Seção 2, apresentamse as considerações principais acerca do método $\mathrm{CCO}$ a título de revisão. Na Seção 3, os resultados obtidos são apresentados e discutidos. Na Seção 4, delineiam-se as conclusões e os potenciais trabalhos futuros. Nas Seções 5 e 6 destacam-se os agradecimentos e as referências bibliográficas.

\section{METODOLOGIA}

A geração de modelos de árvores circulatórias no contexto do método $\mathrm{CCO}$ é baseada nas seguintes considerações (SCHREINER; BUXBAUM, 1993; QUEIROZ, 2013):

- O princípio subjacente à construção da topologia das árvores circulatórias é o de minimização do volume intravascular da árvore:

$V=\pi \sum_{i=1}^{K_{\text {tot }}} l_{i} r_{i}^{2}$

onde $r_{i}$ é o raio do segmento $i, l_{i}$ é o comprimento do segmento $i, K_{t o t}$ é o número de segmentos na árvore em estágio de crescimento;

- A perfusão do domínio de interesse acontece através de um único segmento raiz;

- As porções de tecido ou órgãos a serem fornecidos com sangue são modelados por domínios de perfusão convexos em duas ou três dimensões (2D/3D);

- A árvore circulatória é representada por uma rede de ramificação binária de tubos rígidos cilíndricos (representando os segmentos de vaso), através dos quais escoa um fluido em regime laminar e estacionário;

- A partir de um único segmento de alimentação (segmento raiz), a árvore sucessivamente bifurca até o nível pré-arteriolar, onde é truncada na forma de segmentos terminais. Tais segmentos fornecem sangue à rede microcirculatória (que não é modelada em detalhe) e a quantidade deles, denotada por $N_{t e r m}$, é um dado de entrada para o método $\mathrm{CCO}$.

- O sangue é assumido ser um fluido incompressível, homogêneo e newtoniano; 
- Existe uma lei de potência com expoente constante $\gamma$ que governa a bifurcação dos segmentos na árvore derivada da análise morfométrica de árvores coronárias reais (SHERMAN, 1981), a qual é expressa por

$r_{i}^{\gamma}=r_{e s q}^{\gamma}+r_{d i r}^{\gamma}$,

com um expoente $\gamma \in[2.55 ; 3]$ constante durante a geração do modelo de árvore circulatória;

- A resistência hidrodinâmica $R_{i}$ de um segmento $i$ da árvore é dada pela lei de Poiseuille como segue:

$R_{i}=\left(\frac{8 \eta_{i}}{\pi}\right) \frac{l_{i}}{r_{i}^{4}}$

onde a viscosidade sanguínea $\eta_{i}$ é assumida como sendo constante $\left(\eta_{i}=3.6 \mathrm{cP}\right)$. Logo, o fluxo sanguíneo na microcirculação não é modelado em detalhe pelo método CCO.

- A queda de pressão $\Delta p_{i}$ ao longo de cada segmento $i$ é dada por $\Delta p_{i}=R_{i} Q_{i}$ em que $Q_{i}$ representa o fluxo através do segmento $i$.

- Na posição proximal $\mathbf{x}_{\text {prox }}$ do segmento raiz são impostos o fluxo de perfusão $Q_{p e r f}$ e a pressão de perfusão $p_{\text {perf }}$.

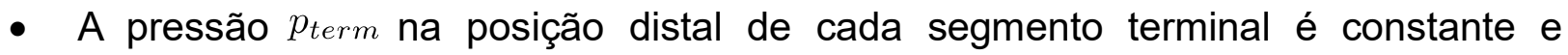
assumida ser a pressão de entrada na região microcirculatória.

- A queda de pressão total $\Delta p$ da árvore é dada por $\Delta p=p_{\text {perf }}-p_{\text {term }}$.

\section{RESULTADOS E DISCUSSÃO}

Nesta seção, apresentam-se os resultados da geração de árvores circulatórias dentro de diferentes domínios convexos 3D: esférico (denotado por F4), elipsoidal (F5), cúbico (F6) e paralelepipédico (F7). Estes domínios possuem o volume de $100 \mathrm{~cm}^{3}$. O objetivo é estudar as características das árvores geradas automaticamente em regiões de diferente geometria. Os dados adotados para construir estas árvores circulatórias foram, segundo o proposto em (KARCH et al., 1999): (i) $\gamma \in\{2.55,3\}$, (ii) $N_{t e r m}=4000$, (iii) $p_{\text {term }}=60 \mathrm{mmHg}$, (iv) fluxo terminal $Q_{\text {term }}^{j}=0.125 \mathrm{ml} / \mathrm{min}$, (v) $p_{\text {perf }}=100 \mathrm{mmHg}$, (vi) $Q_{\text {perf }}=500 \mathrm{ml} / \mathrm{min}$. O centro geométrico destes domínios é a origem do sistema de coordenadas cartesianas. As posições proximais $\mathbf{x}_{\text {iraiz }}[\mathrm{cm}]$ do segmento raiz foram 
fixadas na borda dos domínios F4, F5, F6 e F7 em (0, 0, 0.0288), (0, 0, 0.0181),$(-0.0232$, $0,0.0232)$ e $(-0.0585,0,0.0146)$, respectivamente. Para o domínio $F 5$, a razão entre os semieixos maior e menor é 4 . Para o domínio $\mathrm{F} 7$, a razão entre o comprimento e a altura é 4 .

Para cada domínio de perfusão, o método CCO foi executado 10 vezes para cada valor de $\gamma$ escolhido, os quais foram 2.55 e 3 . Em cada execução, diferentes sequências de números pseudoaleatórios para produzir as posições distais dos segmentos terminais.

$\mathrm{Na}$ Tabela 1 são apresentados os resultados morfométricos para os modelos de árvores circulatórias gerados. Nota-se nesta tabela que o raio do segmento raiz $\left(r_{\text {iraiz }}\right)$ de cada árvore foi maior quando $\gamma=2.55$. O nível de bifurcação máximo $\left(n_{\max }\right)$ foi maior na árvore circulatória gerada dentro do domínio F7.

Tabela 1 - Resultados obtidos empregando o método CCO em domínios 3D arbitrários convexos

\begin{tabular}{|c|c||c|c|c|c|}
\hline$\gamma$ & Domínio & $r_{\text {iraiz }}[\mathrm{mm}]$ & $r_{\min }[\mathrm{mm}]$ & $V\left[\mathrm{~mm}^{3}\right]$ & $n_{\max }$ \\
\hline & F4 & $1.6594 \pm 0.0006$ & $0.0255 \pm 0.0009$ & $821.6331 \pm 3.2723$ & $51 \pm 1$ \\
2.55 & F5 & $1.6645 \pm 0.0009$ & $0.0264 \pm 0.0011$ & $871.7516 \pm 6.6050$ & $62 \pm 2$ \\
& F6 & $1.6662 \pm 0.0018$ & $0.0255 \pm 0.0009$ & $877.3187 \pm 7.4582$ & $56 \pm 3$ \\
& F7 & $1.6783 \pm 0.0014$ & $0.0260 \pm 0.0010$ & $1110.8428 \pm 7.3574$ & $83 \pm 3$ \\
\hline & F4 & $1.2080 \pm 0.0007$ & $0.0393 \pm 0.0011$ & $734.8567 \pm 2.5487$ & $56 \pm 3$ \\
3 & F5 & $1.2176 \pm 0.0016$ & $0.0387 \pm 0.0015$ & $773.2892 \pm 6.4879$ & $70 \pm 3$ \\
& F6 & $1.2196 \pm 0.0017$ & $0.0386 \pm 0.0015$ & $779.5592 \pm 6.5657$ & $61 \pm 2$ \\
& F7 & $1.2583 \pm 0.0013$ & $0.0388 \pm 0.0013$ & $958.0598 \pm 6.7416$ & $91 \pm 2$ \\
\hline
\end{tabular}

Fonte: QUEIROZ (2013).

Na Figura 1, as árvores circulatórias geradas pelo método CCO empregado para vascularização dos domínios F4, F5, F6 e F7 são mostradas. Percebe-se nesta figura que diferentes formas do domínio de perfusão conduzem a diferentes padrões de ramificação, evidenciando a importância da definição geométrica do domínio anatômico a ser perfundido na morfologia final da árvore que o vasculariza. Entretanto, a escolha do valor de expoente de bifurcação praticamente não afetou a estrutura geométrica dos modelos. 
Figura 1 - Vascularização de diferentes domínios 3D e valores de expoente de bifurcação.

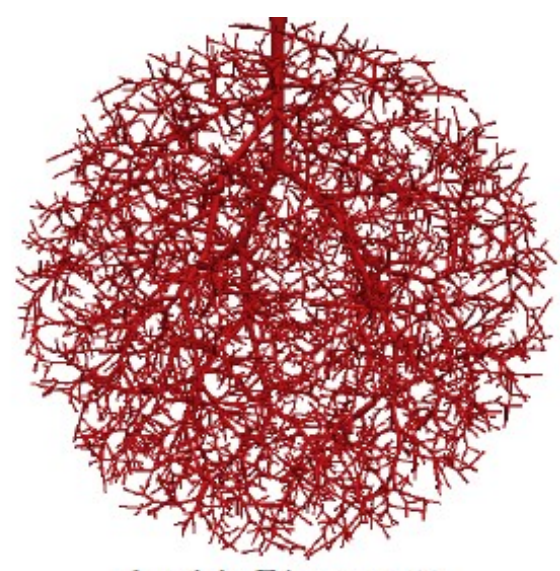

domínio F4 e $\gamma=2.55$

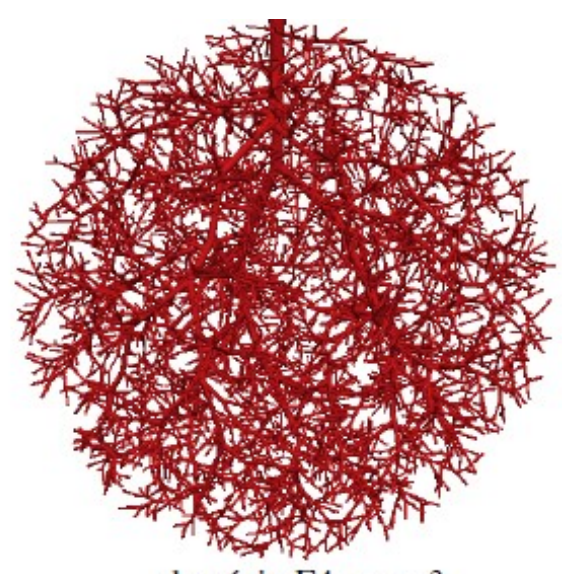

domínio F4 e $\gamma=3$

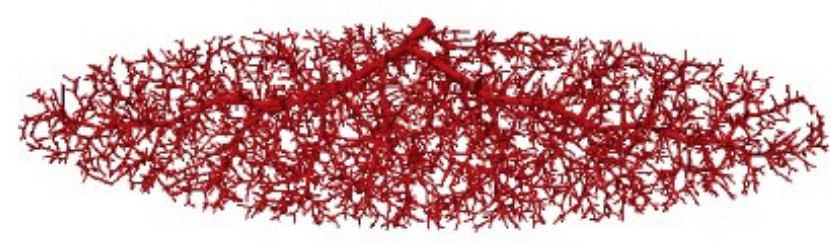

domínio F5 e $\gamma=2.55$

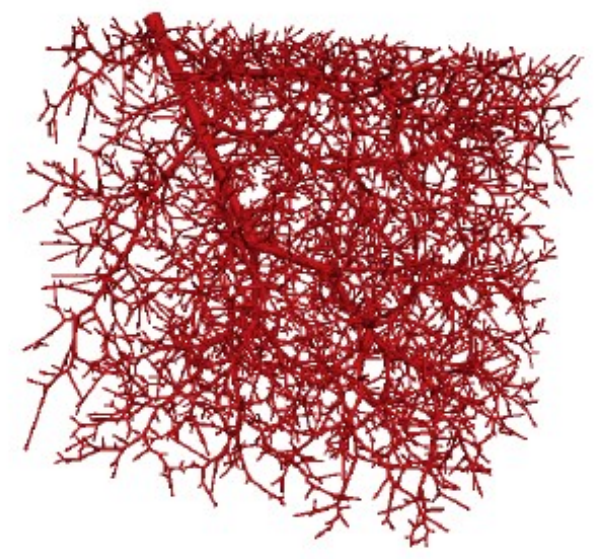

domínio F6 e $\gamma=2.55$

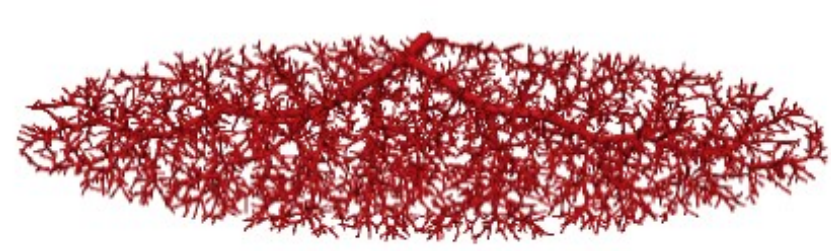

domínio F5 e $\gamma=3$

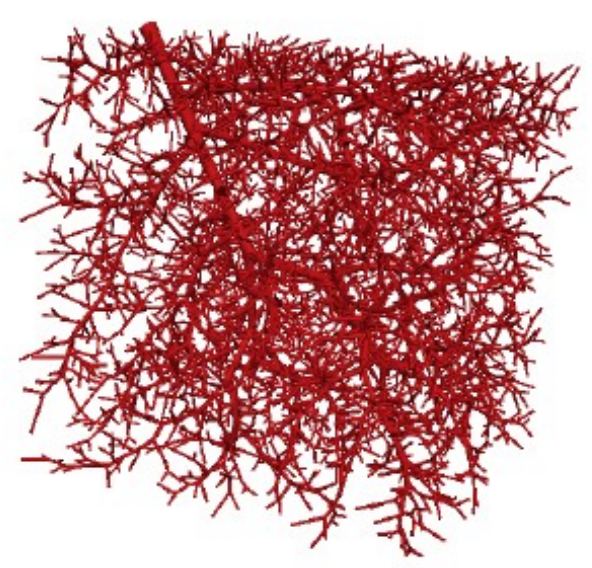

domínio F6 e $\gamma=3$

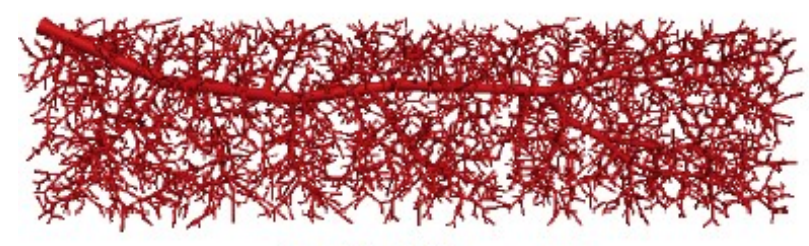

domínio F7 e $\gamma=2.55$

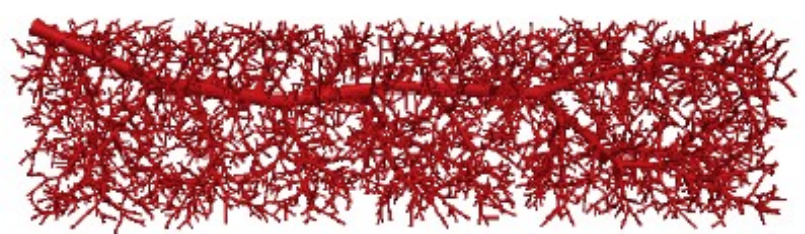

domínio F7 e $\gamma=3$

Fonte: elaboração própria. 


\section{CONCLUSÕES}

Este trabalho apresenta resultados obtidos com aplicação do método $\mathrm{CCO}$ em diferentes domínios 3D. Pelos resultados alcançados, observa-se que a escolha do domínio afeta o volume intravascular total da árvore e nível de bifurcação máximo. Além disso, a escolha do expoente de bifurcação impacta no valor do raio do segmento raiz.

Como perspectivas de trabalhos futuros, vislumbramos as seguintes atividades de pesquisa:

- Comparar morfometricamente os modelos de árvores circulatórias gerados com outras árvores arteriais reais oriundas de corrosão vascular, por exemplo;

- Empregar imagens médicas das redes microcirculatórias de forma a introduzir as particularidades destas redes em cada órgão dentro do processo de vascularização automática;

- Adaptar o método CCO para torná-lo capaz de construir árvores circulatórias contemplando o evento de anastomose dos segmentos.

\section{AGRADECIMENTOS}

Os autores agradecem o apoio financeiro das agências de fomento CNPq e FAPEMIG durante a realização deste projeto.

\section{REFERÊNCIAS}

BUI, A.V.; MANASSEH, R.; LIFFMAN, K.; SUTALO, I. D. Development of optimized vascular fractal tree models using level set distance function. Medical Engineering \& Physics, v. 32, n.7, p. 790-794, 2010.

KARCH, R.; NEUMANN, F.; NEUMANN, M.; SCHREINER, W. A tree-dimensional model for arterial tree representation, generated by constrained constructive optimization. Computers in Biology and Medicine, v. 29, n.1, p. 19-38, 1999.

MATES, R. E.; KLOCKE, F. J.; CANTY, J. M. Coronary capacitance. Progress in Cardiovascular Diseases, v. 31, n.1, p. 1-15, 1988.

ONUKI, T.; NITTA, S. Computer simulation of geometry and hemodynamics of canine pulmonary arteries. Annals of Biomedical Engineering, v. 21, n. 2, p. 107-115, 1993.

QUEIROZ, R.A.B. Construção automática de modelos de árvores circulatórias e suas aplicações em hemodinâmica computacional. 2013. 196 f. Tese (Doutorado em 
Modelagem Computacional) - Laboratório Nacional de Computação Científica, PetrópolisRJ.

SHERMAN, T. F. On connecting large vessels to small. The meaning of Murray's law. The Journal of General Physiology, v. 78, n. 4, p. 431-453, 1981.

SCHREINER, W.; BUXBAUM, P.F. Computer-optimization of vascular trees. IEEE Transactions on Biomedical Engineering, v. 40, n. 5, p. 482-491, 1993.

SCHREINER, W.; NEUMANN, M.; NEUMANN, F.; ROEDLER, S. M.; END, A.; BUXBAUM, P.; MULLER, M.R.; SPIECKERMANN, P. The branching angles in computer-generated optimized models of arterial trees. The Journal of General Physiology, v. 103, n. 6, p. 975-989, 1994.

SCHREINER, W.; NEUMANN, F.; NEUMANN, M.; END, A.; ROEDLER, S. M.; AHARINEJAD, $S$. The influence of optimization target selection on the structure of arterial tree models generated by constrained constructive optimization. The Journal of General Physiology, v. 106, n. 4, p. 583-599, 1995.

SCHREINER, W.; KARCH, R.; NEUMANN, M.; NEUMANN, F.; SZAWLOWSKI, P.; ROEDLER, S. Optimized arterial trees supplying hollow organs. Medical Engineering \& Physics, v. 28, n. 5, p. 416-429, 2006.

VAN BEEK, J.H.; ROGER, S.A.; BASSINGTHWAIGHTE, J.B. Regional myocardial flow heterogeneity explained with fractal networks. American Journal of Physiology, v. 257, p. H1670-H1680, 1989. 